\title{
Influence of Surface Defects on Performance of Journal Bearing
}

\author{
Wenfeng Yin ${ }^{1}$, Sanxing Zhao ${ }^{1 *}$ and Yong Zheng ${ }^{1}$ \\ ${ }^{1}$ School of Machinery and Automation, Wuhan University of Science and Technology, 947\# Heping \\ Avenue, Qingshan District, Wuhan 430081, P.R.China \\ *Corresponding author: zhaosanxing@hotmail.com
}

\begin{abstract}
Keywords: Journal Bearing; Rectangular Defects; Mass Conservation Algorithm; Load Capacity;
\end{abstract} Coefficient of Friction.

\begin{abstract}
This study mainly uses the mass conservation algorithm to analyze the influence of the rectangular surface defects, which are distributed on the bearing surface, in terms of the position of surface defects, defect area, defect depth, and defect occupancy rate on the performance of journal bearing at a steady state. The numerical results show that the rectangular surface defects exert an important influence on the load capacity and friction force. Most of the rectangular surface defects are unfavorable for bearing lubrication, although in some cases, the rectangular surface defects can reduce friction force, they can also weaken load capacity and increase coefficient of friction.
\end{abstract}

\section{Introduction}

In the process of manufacturing and using, it isn't guaranteed that the bearing surface is in the absolute ideal state. For example, references [1,2] pointed out that friction, wear and cavitation erosion will cause pitting, pits, cracks and other defects on the bearing surface in these processes. Scholars have done some researches on the journal bearing with surface defects. The influence of cylindrical surface defects on load capacity and coefficient of friction is analyzed by using the average flow model and the micro convex contact theory in reference [3]. The influence of surface defects with different forms or parameters on the performance of bearing has been analyzed by using the Reynolds boundary condition in [4]. Reference [5] uses FLUENT software to explore the influence of the surface scale-shaped and spherical pits on the drag reduction effect.

There are two important points on surface defects, needed to be mentioned in this study. Firstly, the Reynolds boundary condition was usually used, meeting the conditions: and at the oil film boundary. In the process of iterative calculation, the method of replacing negative pressure with zero is adopted. The method is simple and easy to implement, but the principle of mass conservation cannot be satisfied, and it can only be used in determining of the oil film rupture position and shouldn't be employed to analyze the location of the oil film reformation. Therefore a large number of literatures ${ }^{[6,7,8]}$ pointed out that the mass conservation algorithm of oil film in considering cavitation, such as P- $\theta$ model ${ }^{[9,10]}$, is more in line with the actual situation. Secondly, in practice, many parameters of surface defects will influence performance of bearing, but the previous researches are usually only simple analyses of the surface defects on the performance with limited parameters varying, and are not comprehensive enough. In this study, the influences of the position of surface defects, defect area, defect depth and its occupancy rate on the performance of the journal bearing are studied. Some mechanism of these effects is explained by the P- $\theta$ model.

\section{Brief Introduction of P- $\theta$ Model and its Solution}

Elrod-Adams put forward the $P-\theta$ model on the basis of mass conserving boundary conditions. The whole area is divided into complete lubrication film area and cavitation area. Introducing fluid ratio variable $\theta=\rho_{\mathrm{d}} \rho, \rho_{\mathrm{c}}$ is the average density of gaseous and liquid lubricant in the cavitation area, $\rho$ is the density of lubricate in the oil film zone. In the oil film zone, oil film pressure $P \geq 0$, set $\theta=1$. In the cavitation zone, $P=P_{\mathrm{c}}$, set $0<\theta<1$. In oil film region and cavitation region pressure can be expressed 
by a modified Reynolds equation, and cavitation region's location and area are determined by solving $\theta$.

In this paper, the modified Reynolds equation is following ${ }^{[7,8]}$

$$
\frac{1}{\mathrm{R}^{2}} \frac{\partial}{\partial \varphi}\left(\frac{h^{3}}{12 \eta} \frac{\partial P}{\partial \varphi}\right)+\frac{\partial}{\partial y}\left(\frac{h^{3}}{12 \eta} \frac{\partial P}{\partial y}\right)=\frac{\omega}{2} \frac{\partial(h \theta)}{\partial \varphi} .
$$

In Equation (1), $R$ is radius of journal, $\eta$ is dynamic viscosity of lubricate, $h$ is oil film thickness, $y$ is bearing axial coordinate, $\varphi$ is bearing circumferential angle coordinate, $\omega$ is journal's angular velocity.

By facilitating equation (1), the corresponding dimensionless formula is as follows:

$$
\frac{\partial}{\partial \varphi}\left(H^{3} \frac{\partial \bar{P}}{\partial \varphi}\right)+\left(\frac{2 R}{B}\right)^{2} \frac{\partial}{\partial \lambda}\left(\mathrm{H}^{3} \frac{\partial \bar{P}}{\partial \lambda}\right)=\frac{\partial(\theta H)}{\partial \varphi} .
$$

In the formula (2), $B$ is the width of the bearing. Dimensionless oil film thickness $H=1+\varepsilon \cos \varphi$. Dimensionless oil film pressure $\overline{\mathrm{P}}=P \boldsymbol{C}^{2} / 6 \eta R^{2} \omega$

The mass conservation boundary condition is as follows:

$$
\begin{array}{ll}
p \geq p_{c}, \theta=1 & \text { (Oil film zone) } \\
p=p_{c}, \theta<1 & \text { (Cavitation zone) }
\end{array}
$$

Bearing-end pressure is the atmospheric pressure. The cavitation pressure utilizes experimental value in literature [7]. The solution area is divided into a grid of equal distance. The circumferential and axial directions of the solution area are divided into 360 and 200 nodes respectively, which is a total of 72000 nodes. $p$ and $\theta$ can be solved with over-relaxation iterative method.

In order to verify the correctness of the program, for a specific journal bearing under a certain working condition shown in Table $1, P-\theta$ model has been utilized to receive the calculation results, which has been compared with ones obtained from literature [7] in Table 2.

Table 1 Working Condition and Parameters of Journal Bearing

\begin{tabular}{cc}
\hline Radius clearance [mm] & 0.1455 \\
Bearing radius [mm] & 50 \\
Ratio of length to diameter & 1.333 \\
Angular velocity [rad/s] & 48.1 \\
Dynamic viscosity [pa.s] & 0.0127 \\
Atmospheric pressure [pa] & 0.0 \\
Cavitation pressure [Mpa] & -0.72 \\
Eccentricity & 0.61 \\
\hline
\end{tabular}

In Table 2, the dimensionless load capacity and the attitude angle are listed. It shows that the

\begin{tabular}{|c|c|c|c|}
\hline & Literature [7] & $P-\theta$ model & Error \\
\hline $\begin{array}{l}\text { Dimensionless lo } \\
\text { ad capacity }\end{array}$ & 9.29 & 9.24 & $-0.54 \%$ \\
\hline Attitude angle $\left[{ }^{\circ}\right]$ & 66.59 & 65.96 & $0.95 \%$ \\
\hline
\end{tabular}
results from $P-\theta$ model are in good agreement with the experimental data in literature [7]. So this algorithm is verified reliable.

Table 2 The comparison of the results in [7] and ones from $P-\theta$ model 


\section{Mathematical Model}

A rectangular coordinate system is established as shown in Fig. 1. $R$ and $r$ are the radius of the bearing and journal, $\mathrm{o}$ and $\mathrm{o}_{1}$ are bearing and journal center, $e$ is the eccentric distance, $F$ is an external load, $\varphi$ is measured from where the maximum oil film thickness is.

Rectangular surface defects are distributed over the bearing surface, and the circumferential and axial lengths of each rectangular defect are $a_{1}, a_{2}$. The depth of defect is $h_{\mathrm{p}}$, The surface defects are located in the center of the imaginary control unit, and the control unit lengths are $b_{1}, b_{2}$.

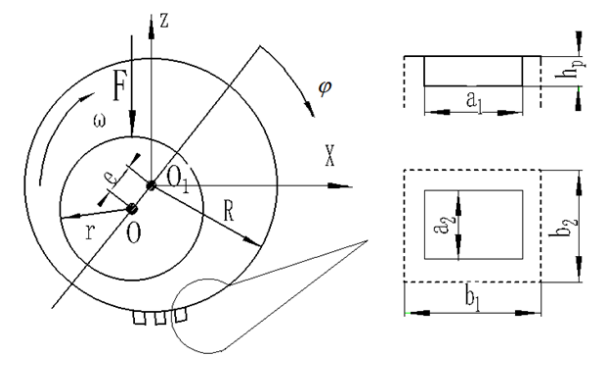

Figure 1. Sketch map of surface defects in journal bearing

Oil film thickness of journal bearing with surface defects is expressed as follows

(a) In non-defect surface region

$h=c+e \cos \varphi$.

(b) In defect surface region

$h=c+e \cos \varphi+h_{p}$.

Oil-film force and oil-film friction force can be computed ${ }^{[7]}$ after pressure distribution has been known. Then coefficient of friction can be easily received.

\section{Results and Analysis}

The bearing's working conditions and geometric parameters are shown in Table 1. In the simulation, in order to facilitate the processing of data, choosing 8 grid size in either direction as a control unit, that is to say , b1 $=6.98 \mathrm{~mm}, \mathrm{~b} 2=5.33 \mathrm{~mm}$, i.e., $45 \times 25$ control units are equally distributed over the bearing surface("unit matrix" by 45 columns with different circumferential coordinates $\varphi$ and 25 rows with different axial coordinate $\lambda$, similarly hereinafter). If possible, each control unit only contains a defect as shown in Fig.1. The influence of the variation on temperature and pressure on the lubricant's viscosity is neglected, and the effects of inlet pressure.

\subsection{Influence of Circumferential Position of Surface Defect Area on Performance of Journal Bearing}

To explore the influence of the different circumferential position of the surface defect area on the performance of the journal bearing, a certain area of $4 \times 25$ rectangular defects uniformly distributed on the bearing surface is used as a case study. For the defect area, the circumferential width spans 4 control units, the axial width is set to $\mathrm{B}(25$ control units). The depth of rectangular defects is set to $0.25 \mathrm{c}, 0.50 \mathrm{c}, 0.75 \mathrm{c}$ respectively. The position of defect area can be defined by the circumferential angle, which can change from $0^{\circ}$ to $360^{\circ}$. The surface defect area is symmetrical with respect to the circumferential middle-section of the bearing. It must be point out that ai=0.5bi $(i=1,2)$, other parameters of the journal bearing remain unchanged.

As showed in Fig. 2(a), there is a great influence on the load capacity of journal bearing with the different circumferential position of surface defect area. When the defect area is located between $30^{\circ}$ and $120^{\circ}$ or between $150^{\circ}$ and $180^{\circ}$, the bearing's load capacity progressively gets smaller, yet it gradually gets larger when the circumferential angle is between $120^{\circ}$ and $150^{\circ}$ or between $180^{\circ}$ and 
$240^{\circ}$, in addition, it basically remains the same and roughly keeps the same with no surface defects $(2183 \mathrm{~N})$ when the position is between $240^{\circ}$ and $330^{\circ}$. When surface defect area is located at $180^{\circ}$, the bearing's load capacity turns to be the smallest, because the surface defects are just near the region with the maximum oil film pressure, the existence of the surface defect is equivalent to increase the oil film thickness, which leads to the oil film pressure decrease. Moreover, the presence of surface defects has a negligible effect on the load capacity when they locate at the unloading region of the bearing $\left(240^{\circ}\right.$ to $\left.300^{\circ}\right)$.

(a)

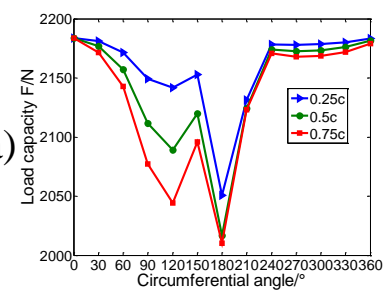

(b)

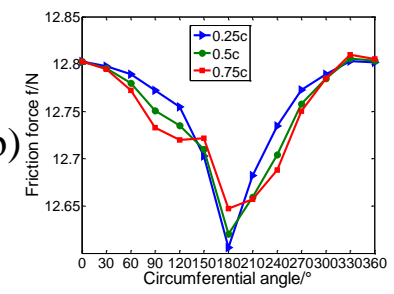

(c)

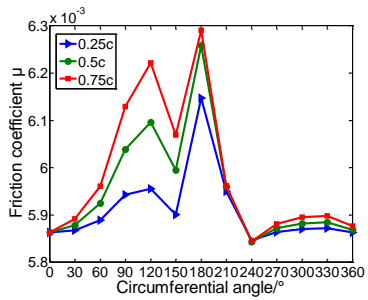

Figure 2. Performance of bearing with different circumferential position of surface defect area

As shown in Fig. 2(b), it can be observed the friction force reduces gradually when the surface defects position angle less than $180^{\circ}$, the tendency is opposite when the position angle greater than $180^{\circ}$. In addition, no matter where the surface defect area is in the circumferential position, the variation of friction force is not large, with its value $<2 \%$. So a conclusion can be made that the friction force almost has nothing to do with the circumferential position of the surface defect.

As shown in Fig. 2(c), the changing rule of the coefficient of friction with different surface defect circumferential position is roughly opposite to the load capacity of journal bearing. Because of the small variation of friction force $(12.6 \mathrm{~N} \sim 12.8 \mathrm{~N})$, the changing of the coefficient of friction is mainly influenced by the changing of the load capacity.

The deeper the surface defect depth, the smaller the load capacity, the larger coefficient of friction. The load capacity is maximum and the friction force is minimum when surface defects' position is located at $240^{\circ}$ and depth is $0.25 \mathrm{c}$, in which circumstance the surface defects have a certain effect of anti-friction. From these results, it shows that most of the rectangular surface defects are unfavorable for the performance of journal bearing, although in some cases, the rectangular surface defects can reduce friction, they can also weaken the load capacity and increase the coefficient of friction.

\subsection{Influence of Circumferential Width of Surface Defect Area on Performance of Journal Bearing}

Suppose that the surface defect area of bearing is symmetrical to the circumferential middle-section of the bearing and the surface defects axial width is set to $B$ and depth is $0.5 c$, other parameters remain unchanged. The circumferential width of surface defect area and its location can be adjusted so as to explore the influence of the circumferential width of surface defect area on the performance of journal bearing. Fig. 3 reflects the influence of circumferential width on the performance of journal bearing, the abscissa 1, 2, 3, 4, 5 in Fig. 3 represent the circumferential range of surface defect area $180^{\circ}-360^{\circ}, 200^{\circ}-360^{\circ}, 220^{\circ}-360^{\circ}, 240^{\circ}-360^{\circ}, 260^{\circ}-360^{\circ}$, respectively.

(a)

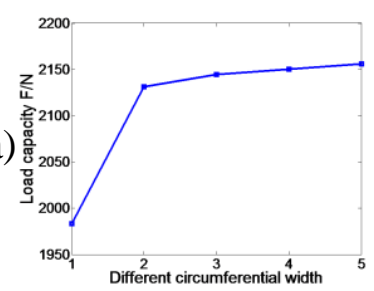

(b)

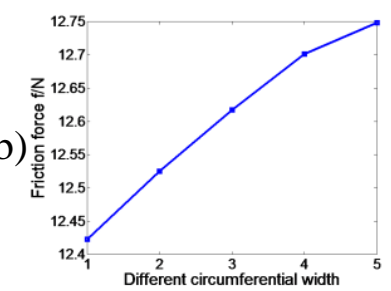

(c)

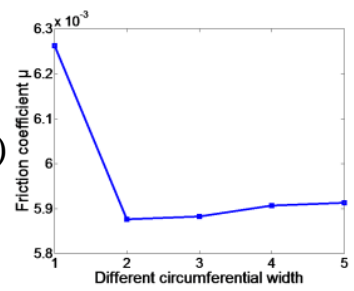

Figure 3. Performance of bearing with different circumferential width of surface defect area 
As showed in Fig. 3, the circumferential width of surface defect area has a great influence on the performance of journal bearing. The load capacity and friction force increase when the starting position of the surface defect area changes from $180^{\circ}$ to $260^{\circ}$, but the change of the load capacity becomes slower after $200^{\circ}$. The coefficient of friction changes relatively quickly when the starting position of the surface defect area changes from $180^{\circ}$ to $200^{\circ}$, and almost remains unchanged after $200^{\circ}$. It indicates that the performance of journal bearing is better than others when it ranges from $260^{\circ}$ to $360^{\circ}$, meanwhile, in which situation the defect area is the least among these 5 cases.

\subsection{Influence of Area Occupancy Rate of the Surface Defect on Performance of Journal Bearing}

To explore the influence of area occupancy rate of the surface defect on the performance of journal bearing, the defects are uniformly distributed over all of the bearing surface, and symmetrical to the circumferential middle-section of the bearing. The depth of the surface defects are set to $0.5 c$. Cases with 3 kinds of defect area occupancy rate have been considered, the performance of journal bearing has been calculated. In Fig. 4, the abscissa 1, 2, 3 mean the surface defect size $a_{\mathrm{i}}=0.25 b_{\mathrm{i}}, a_{\mathrm{i}}=0.5 b_{\mathrm{i}}$, $a_{\mathrm{i}}=0.75 b_{\mathrm{i}}(\mathrm{i}=1,2)$ respectively, and the abscissa 0 means no surface defect.

(a)

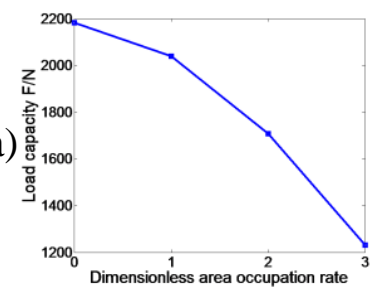

(b)

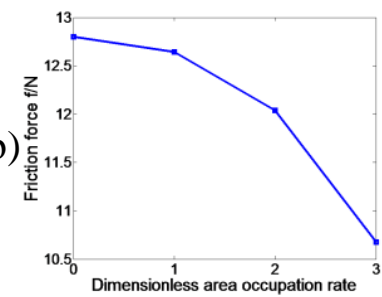

(c)

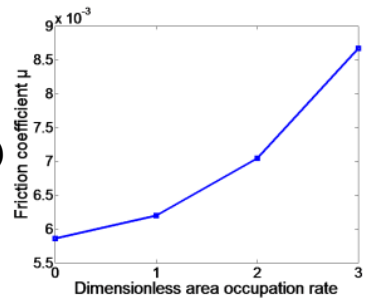

Figure 4. Performance of bearing with different area occupancy rate of surface defect

As showed in Fig. 4, with the increasing of area occupancy rate of surface defect, the load capacity and friction force decrease, and the coefficient of friction increases. The load capacity grows to the maximum value and the coefficient of friction reduces to the minimal value when there is no defect on the bearing surface. In a word, the bigger defect area occupancy rate, the worse the overall performance of journal bearing.

\section{Conclusions}

This paper mainly analyzes the influence of the surface defects on the performance of journal bearing. Based on the mass conservation algorithm, it explores many influence factors, such as position of surface defects, surface defect area, defect depth, area occupancy rate of surface defect. It shows that the surface defects have a very complicated influence on the performance of journal bearing. When analyzing the influence of surface defects, the effects of all these factors should be combined. Some conclusions can be drawn as follows:

Compared with the smooth surface, for defect cases presented in this study, surface defects usually worsen the performance of journal bearing. And the larger defect area, the worse the performance. For a certain area of defects, defects have a comparatively small influence when they are located at the unloaded region of bearing surface. The deeper defect depth, the smaller the load capacity, and the larger the coefficient of friction. Obviously, most of the rectangular surface defects are unfavorable for the performance of journal bearing, although in some cases these surface defects can reduce friction force, they can weaken the load capacity and increase the coefficient of friction.

\section{Acknowledgements}

This work was supported by NSFC (51405350) and partly by the open fund of the Key Laboratory for Metallurgical Equipment and Control of Ministry of Education at Wuhan University of Science and Technology (2014A15). 


\section{References}

[1] Gangzhi Wang, Xiangyin Xu, Xingyu Liang. An influence of journal misalignment on lubrication and wear of main bearings of ICE. Lubrication engineering, 2011, 36(3):49-54.

[2] Qi An. Review of research on cavitation erosion in journal bearing. China mechanical engineering, 2002, 13(3):263-266.

[3] Dumin Lei, Suhua Wang. Effect of surface texture on mixed lubrication characteristics of journal bearings. Bearing, 2013,2:36-39.

[4] Haifeng Hu. A simulation analysis of surface defect effect on radial sliding bearing. Master's thesis, Dalian Maritime University, 2014.

[5] Fengchao Qian. Numerical simulation on drag reduction of bionic squamous cavity non-smooth surface. Master's thesis, Dalian University of Technology. 2013.

[6] Pengqing Qiu, Sheng Cui, Xiaofeng Xu, et al. The comparison between Elrod algorithm and negative pressure filling algorithm. Journal of Vibration and Shock, 1998, 4:12-17.

[7] Hong Su, Xiaojing Wang. Study on oil film cavitation based on mass conservation boundary conditions. Master's thesis, Shanghai University, 2003.

[8] R Ausas, P Ragot, J Leiva, et al. The impact of the cavitation model in the analysis of the micro-textured lubricated journal bearings. Journal of tribology, 2007, 129(4):868-875.

[9] R F Ausas, M Jai, G C Buscaglia. A mass-conserving algorithm for dynamic lubrication problems with cavitation. Journal of Tribology, 2009, 131(1):1-27.

[10] Qiang Li, Shuiying Zheng, Shulian Liu. Analysis of the performance of journal bearing with JFO boundary condition. Mechanical Strength, 2010,32(2) :270-274 